Documentation et bibliothèques

DOCUMENTATION BIBLIOTHËQUES

\title{
Les publications des Commissions sur le processus de détermination de l'avenir politique et constitutionnel du Québec
}

\section{Patrick Cossette}

Volume 40, numéro 2, avril-juin 1994

URI : https://id.erudit.org/iderudit/1033457ar

DOI : https://doi.org/10.7202/1033457ar

Aller au sommaire du numéro

Éditeur(s)

Association pour l'avancement des sciences et des techniques de la documentation (ASTED)

\section{ISSN}

0315-2340 (imprimé)

2291-8949 (numérique)

Découvrir la revue

Citer cet article

Cossette, P. (1994). Les publications des Commissions sur le processus de détermination de l'avenir politique et constitutionnel du Québec.

Documentation et bibliothèques, 40(2), 97-100. https://doi.org/10.7202/1033457ar

Tous droits réservés (c) Association pour l'avancement des sciences et des techniques de la documentation (ASTED), 1994
Ce document est protégé par la loi sur le droit d'auteur. L'utilisation des services d'Érudit (y compris la reproduction) est assujettie à sa politique d'utilisation que vous pouvez consulter en ligne.

https://apropos.erudit.org/fr/usagers/politique-dutilisation/ 


\title{
Les publications des Commissions sur le processus de détermination de l'avenir politique et constitutionnel du Québec
}

\author{
Patrick Cossette * \\ Bibliothèque de l'Assemblée nationale \\ Québec
}

\begin{abstract}
Mises sur pied dans la foulée de la Commission Bélanger-Campeau, la Commission d'étude des questions afférentes à l'accession du Québec à la souveraineté et la Commission d'étude sur toute offre d'un nouveau partenariat constitutionnel laissent derrière elles une abondante documentation sur l'épineux dilemme constitutionnel québécois. L'auteur décrit ici les nombreuses publications produites sous l'égide des deux organismes. Somme toute, la tourmente constitutionnelle de la période 1990 1992 a beau avoir débouché sur une impasse, du moins en reste-t-il de solides munitions pour la prochaine ronde...
\end{abstract}

II y a de ces sagas qui semblent sans fin tant elles font partie du paysage quasi immuable d'une société. La question constitutionnelle québécoise et canadienne, depuis la crise de l'accord du lac Meech en 1990 jusqu'au référendum sur l'accord de Charlottetown en 1992, a connu des rebondissements dignes des meilleurs auteurs de téléromans. Entre ces deux points d'orgue que constituent ces accords désavoués, le gouvernement du Québec a produit une énorme somme de réflexions et d'opinions par l'entremise de commissions parlementaires spéciales.

C'est ce que fit tout d'abord la Commission sur l'avenir politique et constitutionnelle du Québec (mieux connue sous le nom de Commission Bélanger-Campeau) de septembre 1990 à la remise de son rappoit en mars 1991. Documentation et Bibliothèques, dans une livraison précédente, a déjà fait écho aux publications de cette commission ${ }^{1}$. Les recommandations de cette dernière prévoyaient l'adoption par l'Assemblée nationale d'une loi établissant le processus de détermination de l'avenir politique et constitutionnel du Québec. C'est ainsi que fut adopté, le 20 juin 1991 , le projet de loi $150^{2}$, qui fixait la tenue d'un référendum et donnait naissance à deux commissions parlementaires spéciales chargées de poursuivre la démarche déjà entreprise. Dans l'année qui suivit, les travaux respectifs de ces deux commissions jumelles scrutèrent avec force détails les tenants et aboutissants des deux options qui divisent les Québécois depuis longtemps, inscrites dans les noms mêmes de ces commissions, soit "Commission d'étude des questions afférentes à l'accession du Québec à la souveraineté» et "Commission d'étude sur toute offre d'un nouveau partenariat constitutionnel».

Moins couvertes par les médias que leur "commission-mère» (BélangerCampeau), ces commissions n'en ont pas moins livré des publications d'un grand intérêt pour la compréhension de la question constitutionnelle québécoise. II est vrai que la Commission BélangerCampeau s'écartait des pratiques parlementaires, puisqu'elle comptait parmi ses membres des parlementaires fédéraux figurant aux côtés de personnalités publiques non élues. L'attention médiatique a également été sollicitée dans le sillon de la tournée québécoise destinée à entendre les témoignages de tous et chacun. Dans le cas des commissions qui nous occupent, seuls y siégeaient des députés de l'Assemblée nationale, et le mode de fonctionnement s'est déroulé en majeure partie sur la base d'invitations auprès d'experts. Le produit de tous ces travaux mérite un tour d'horizon.

Commission d'étude des questions afférentes à l'accession du Québec à la souveraineté

\section{États de la question}

Une série de 19 fascicules intitulée L'avenir politique et constitutionnel du Québec synthétise les «têtes d'affiche thématiques» sur lesquelles les commissaires devaient se pencher. Produites par l'équipe du Secrétariat commun aux deux commissions, ces synthèses ont eu pour fonction d'éclairer les commissaires sur les questions abordées par les experts invités et les études commandées. Parcourues de balises chronologiques et documentaires, elles brossent un portrait des aspects juridiques et économiques de l'accession du Québec à la souveraineté. Elles sont toutes dotées d'un plan identique: introduction, questions ou problèmes traités, analyse sommaire des questions soulevées, recommandations et, dans certains cas, des annexes. D'une indéniable qualité quoique peu volumineuses, on peut déplorer cependant que, dans ce qui se veut une série, on n'ait pas établi de numérotation unificatrice ni d'uniformité au niveau des intitulés des sous-séries ${ }^{3}$ (l'intention y était au départ mais n'a pu, semble t-il, être réalisée). La série est complétée par les biographies des commissaires, une description des activités des commissions parlementaires fédérale et provinciales qui se déroulaient au même moment et un aperçu des positions du Québec en matière constitutionnelle jusqu'à 1991. Tous ces textes ont été traduits en anglais.

* Patrick Cossette était le documentaliste du Secrétariat des Commissions sur le processus de détermination de l'avenir politique et constitutionnel du Québec.

1. Gaston Bernier, «La Commission BélangerCampeau et ses publications", Documentation et Bibliothèques, vol. 37, no 4 (octobre-décembre 1991), 137-140.

2. Loi sur le processus de détermination de l'avenir politique et constitutionnel du Québec, L.Q. 1991, chap. 34.

3. Voir annexe. 


\section{Exposés et études}

Les travaux de la Commission ont été consacrés essentiellement aux témoignages et aux recherches des experts invités à livrer leurs réflexions. Les résultats de leurs analyses sont publiés dans quatre recueils de textes totalisant plus de 3000 pages et divisés en deux grand blocs: les attributs d'un Québec souverain et ses implications. Chacun des quatre volumes est lui-même divisé en thèmes autour desquels on a regroupé les 67 études. Un index général des sujets permet un accès par descripteurs. L'éventail. des thèmes couverts, la notoriété des auteurs (plusieurs sont de réputation internationale) et le caractère inédit de plusieurs de ces études en font la plus importante somme de connaissances jamais produite sur le sujet.

II convient d'indiquer que seuls les documents déposés par les experts ou encore commandés auprès de ceux-ci sont repris dans les Exposés et études. Dans les autres cas, la référence au Journal des débats ou à une oeuvre précédemment publiée dirige le lecteur vers une autre source. Le Secrétariat a également choisi de soustraire de cette publication les textes soumis par les représentants politiques des nations autochtones. Leurs présentations faisaient suite à une requête spéciale des membres de la Commission et s'écartaient du plan de travail préétabli. Ces textes sont toutefois disponibles à la Bibliothèque de l'Assemblée nationale, comme le sont les versions originales de ceux figurant dans les recueils. Certains textes d'auteurs anglophones ont été traduits, mais il en demeure quatre qui ne l'ont malheureusement pas été.

\section{Comptes rendus intégraux}

Du 28 août 1991 au 16 septembre 1992, la Commission a tenu 38 séances publiques et entendu 53 spécialistes, dont l'intégralité des propos est consignée au Journal des débats de la Commission. Comme en avait bénéficié auparavant la Commission Bélanger-Campeau, la Direction du Journal des débats de l'Assemblée nationale a accordé à la commission spéciale un traitement différent de celui offert aux commissions parlementaires permanentes en créant une pagination et un index détaillé propres à ses débats.
Le projet de rapport

Aucune des deux commissions spéciales n'a officiellement terminé son mandat. Après plus d'une année de travaux intenses, et bousculés par l'échéancier politique, les commissaires décidèrent en septembre 1992 de rendre public le projet de rapport rédigé par le Secrétariat. II ne s'agit donc pas d'un document approuvé par les parlementaires avec recommandations à l'appui comme l'exigeait la loi, mais d'une synthèse de 193 pages reprenant les lignes de force des analyses des experts et résumant leurs points de vue. Découpé thématiquement de la même manière que la série Exposés et études, il s'apparente à ce qu'un journaliste a appelé «un véritable manuel d'instructions pour la souveraineté ${ }^{4} »$. Une version anglaise a également été publiée.

Commission d'étude sur toute offre d'un nouveau partenariat de nature constitutionnelle

Comme sa contrepartie, cette commission n'a jamais officiellement terminé ses travaux et se trouve ajournée sine die. Les chercheurs intéressés doivent se contenter des textes déposés par les experts invités ${ }^{5}$ et de la transcription des débats. On y retrouve les propos recueillis au cours des 27 séances publiques tenues entre le 28 août 1991 et le 10 septembre 1992, avec index à l'appui. Les événements politiques ont divisé les travaux de cette commission en deux temps. Jusqu'à la conclusion de l'entente de Charlottetown, les experts sont venus commenter devant la Commission les propositions déjà divulguées par le gouvernement fédéral, à la lumière des demandes traditionnelles du Québec. Puis, pressées par l'échéancier du référendum, les dernières séances ont porté sur l'analyse de l'accord de Charlottetown proprement dit, sans toutefois déboucher sur des recommandations.

Sil'on excepte trois des fascicules de la série L'avenir politique et constitutionnel du Québec (dont deux d'ailleurs sont communs aux deux commissions), le Secrétariat n'a rien produit sous l'autorité de cette dernière.

\section{Articles de journaux}

En marge des travaux des commissions, la Division de la documentation de presse de la Bibliothèque de l'Assemblée nationale a pris l'initiative de lancer une série de recueils d'articles de journaux sur le débat constitutionnel. Cette compilation se présente en deux cahiers couvrant la période d'août 1991 à septembre $1992^{6}$. Les articles de tous les quotidiens québécois ayant porté sur le sujet y ont été regroupés en quatre thèmes: propositions fédérales, questions afférentes à la souveraineté, éditoriaux et analyses et sondages d'opinion. Des recueils semblables ont également été produits sur la Commission Bélanger-Campeau et le référendum sur l'accord de Charlottetown ${ }^{7}$.

\section{Conclusion}

Avec les travaux de ces deux commissions s'est achevé un épisode de la saga constitutionnelle québécoise qui avait commencé par la Commission BélangerCampeau. Dans un de ces retours de pendule dont l'histoire a le secret, il est fort possible que l'on revoie les travaux de celles-ci dans l'actualité dans un avenir plus ou moins rapproché.

Par l'abondance et la qualité des publications, les trois commissions laissent derrière elles un remarquable héritage documentaire. Un honnête souci de transparence et de diffusion de l'information a

4. Jean-Hugues Roy, «Commission sur la souveraineté: invitation au voyage», Voir (Montréal), vol. 6, no 2 (29 octobre 1992), 8-10.

5. Disponibles à la Bibliothèque de l'Assemblée nationale.

6. Le débat constitutionnel: articles de journaux, Août 1991-janvier 1992 (2 cahiers); Janvier 1992-septembre 1992 (2 cahiers), Québec, Bibliothèque de l'Assemblée nationale, Division de la documentation de presse, 1992.

7. La Commission Bélanger-Campeau: articles de journaux, juin 1990-juin 1991, Québec, Bibliothèque de l'Assemblée nationale, Division de la documentation de presse, 1991. (Cahier 1: Gouvernement du Québec, travaux de la Commission et extraits de mémoires; Cahier 2: Éditoriaux et analyses); Le référendum du 26 octobre 1992: articles de journaux, septembre 1992-décembre 1992, Québec, Bibliothèque de l'Assemblée nationale, Division de la documentation de presse, 1993. 
semblé inspirer leurs activités ${ }^{8}$. II s'agit là d'une préoccupation qu'il serait souhaitable de retrouver à la grandeur du domaine des publications et parapublications gouvernementales, encore trop mené par l'arbitraire. À ce sujet, les publications des commissions royales, des commissions d'enquête et des commissions parlementaires spéciales restent souvent exemplaires. On peut y voir le fruit du caractère éminemment public de leurs mandats et d'une concentration exceptionnelle de ressources réunies pour une période de temps limitée.

Annexe: Publications des Commissions sur le processus de détermination de l'avenir politique et constitutionnel du Québec'

\section{1- Composition des commissions}

Les commissions parlementaires surl'avenir politique et constitutionnel du Québec. Québec, Assemblée nationale, 18 octobre 1991. 3 p., 1 annexe.

Composition des deux commissions et biographies de leurs membres et des dirigeants du Secrétariat. Document publié dans la série L'avenir politique et constitutionnel du Québec

\section{2- Commissions fédérale et provinciales}

Commissions ou comités créés dans les autres provinces canadiennes et au fédéral. Québec, Assemblée nationale, 30 octobre 1991. 21 p.; 20 novembre 1991. 19 p.

Déroulement des activités des autres instances fédérale et provinciales en date des mois d'octobre et de novembre 1991. Documents publiés dans la série L'avenir politique et constitutionnel du Québec par le Secrétariat de la Commission d'étude sur toute offre d'un nouveau partenariat de nature constitutionnelle.

3- Publications du Secrétariat de la Commission d'étude des questions afférentes à l'accession du Québec à la souveraineté

Projet de rapport: document de travail. Québec, Assemblée nationale, 1992.193p.

\section{a) Série Exposés et études}

Les attributs d'un Québec souverain. Québec, Assemblée nationale, 1992. 921 p. (Exposés et études; volume 1)

Découpage du volume: Une collectivité nationale distincte. Les droits de la personne et des minorités. Les nations autochtones. Le territoire. Les institutions. La personnalité internationale.

Les implications de la mise en oeuvre de la souveraineté: les aspects juridiques, les services gouvernementaux. Québec, Assemblée nationale, 1992. 427 p. (Exposés et études; volume 2)

Principaux thèmes abordés: continuité juridique, services fédéraux, défense.

Les implications de la mise en oeuvre de la souveraineté: les aspects économiques et les finances publiques (Première partie). Québec, Assemblée nationale, 1992. 493 p. (Exposés et études; volume 3 )

Principaux thèmes abordés: relations économiques et commerciales, agriculture, textile et vêtement, automobile, Communauté européenne.

Les implications de la mise en oeuvre de la souveraineté: les aspects économiques et les finances publiques (Deuxième partie). Québec, Assemblée nationale, 1992. 831 p. (Exposés et études; volume 4)

Principaux thèmes abordés: régions frontalières, services fédéraux, monnaie, secteur financier, conséquences économiques, actif et dette, finances publiques.

b) Série L'avenir politique et constitutionnel du Québec ${ }^{10}$

La démarche du Québec. Québec, Assemblée nationale, 20 novembre 1991. 7 p.

L'accession à la souveraineté: le processus. Québec, Assemblée nationale, 8 octobre 1991. $9 \mathrm{p}$.

L'accession à la souveraineté: la déclaration de souveraineté. Québec, Assemblée nationale, 4 novembre 1991. 9 p., 4 annexes.
L'accession à la souveraineté: la reconnaissance internationale. Québec, Assemblée nationale, 4 novembre 1991. 9 p.

L'accession à la souveraineté: l'immigration et la question des réfugiés. Québec, Assembléenationale, 14 novembre 1991.9p.

L'accession à la souveraineté: les options monétaires d'un Québec souverain. Québec, Assemblée nationale, 25 novembre 1991. 11 p., 1 annexe.

L'accession à la souveraineté: les droits des minorités. Québec, Assemblée nationale, 26 novembre 1991. 9 p.

L'accession à la souveraineté: l'élaboration d'une constitution. Québec, Assemblée nationale, 12 décembre 1991. 9 p., 1 annexe.

La déclaration de souveraineté: les frontières d'un Québec souverain. Québec, Assemblée nationale, 11 octobre 1991. 10 p., 1 annexe.

La succession d'États: la succession aux traités. Québec, Assemblée nationale, 18 décembre 1991. $9 \mathrm{p}$.

Succession d'États: la nationalité. Québec, Assemblée nationale, 14 janvier $1992.9 \mathrm{p}$.

Succession d'États: la défense. Québec, Assemblée nationale, 15 janvier $1992.10 \mathrm{p}$.

La succession d'États: les relations internationales d'un Québec souverain. Québec, Assemblée nationale, 11 février 1992.11p.

8. Le Secrétariat des Commissions s'est notamment efforcé de distribuer le projet de rapport et les exposés et études aux bibliothèques publiques, collégiales et universitaires du Québec, ainsi qu'à un grand nombre de citoyens et d'organismes.

9. Le projet de rapport, les volumes 2,3 et 4 des exposés et études, ainsi que les comptes rendus des séances publiques sont toujours disponibles auprès du Service de distribution des documents parlementaires de l'Assemblée nationale.

10. Voir également les parties 1 et 2. 
Partenariat économique: les relations commerciales d'un Québec souverain. Québec, Assemblée nationale, 16 janvier 1992. $14 \mathrm{p}$.

Les relations entre l'État et les nations autochtones. Québec, Assemblée nationale, 7 février 1992. 23 p., 4 annexes.

Les implications sociales et culturelles de la souveraineté: bref portrait démographique, culturel et social du Québec contemporain. Québec, Assemblée nationale, 14 février 1992. $16 \mathrm{p}$.

Les régions frontalières: l'Outaouais québécois. Québec, Assemblée nationale, 17 février 1992. $9 \mathrm{p}$.

\section{LA SOURCE QUÉBÉCOISE D'INFORMATION SUR LES LOGICIELS}

\section{L'outil de recherche indispensable!}

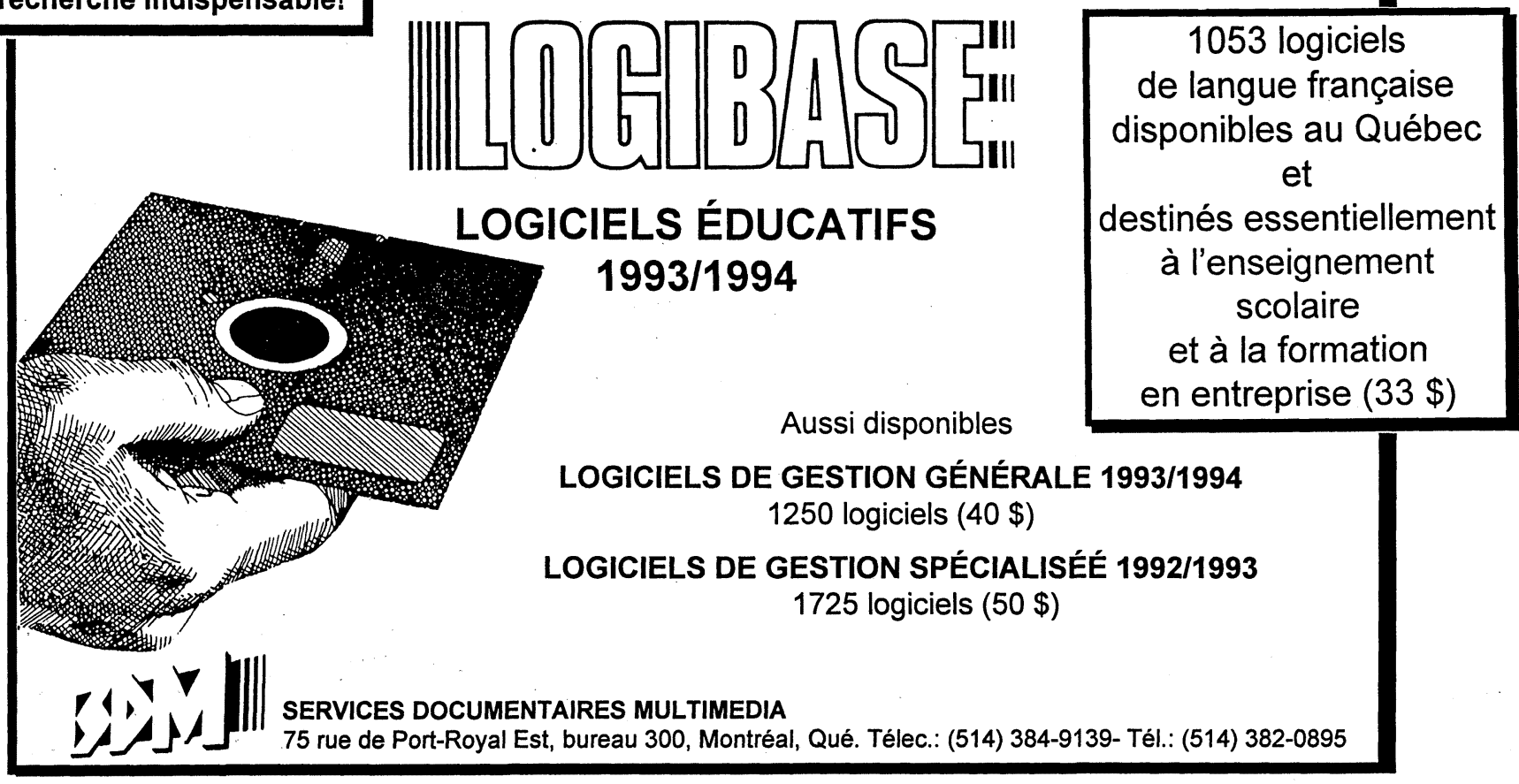

